\title{
Effects of the wave function localization in AllnGaN quaternary alloys
}

\author{
Fei Wang, Shu-Shen Li, and Jian-Bai Xia \\ of Sciences, P.O. Box 912, Beijing 100083, China \\ H. X. Jiang and J. Y. Lin \\ Department of Physics, Kansas State University, Manhattan, Kansas 66506
}

State Key Laboratory for Superlattices and Microstructures, Institute of Semiconductors, Chinese Academy

Jingbo Li and Su-Huai Weia)

National Renewable Energy Laboratory, Golden, Colorado 80401

(Received 15 May 2007; accepted 19 July 2007; published online 10 August 2007)

\begin{abstract}
Using the first-principles band-structure method and the special quasirandom structures approach, the authors have investigated the band structure of random $\mathrm{Al}_{x} \mathrm{In}_{y} \mathrm{Ga}_{1-x-y} \mathrm{~N}$ quaternary alloys. They show that the wave functions of the band edge states are more localized on the InN sites. Consequently, the photoluminescence transition intensity in the alloy is higher than that in GaN. The valence band maximum state of the quaternary alloy is also higher than $\mathrm{GaN}$ with the same band gap, indicating that the alloy can be doped more easily as p-type. (C) 2007 American Institute of Physics. [DOI: 10.1063/1.2769958]
\end{abstract}

Group III-nitride semiconductors, AlN, GaN, and InN, and their ternary alloys AlGaN, GaInN, and AlInN, have been studied extensively because their band gaps cover a wide range from 0.7 to $6.1 \mathrm{eV},{ }^{1}$ which makes them suitable for a variety of applications such as blue and ultraviolet (UV) light-emitting diodes, laser diodes, and high-temperature/ high-power heterostructure field-effect transistors. ${ }^{2}$ Recently, the quaternary alloys $\mathrm{Al}_{x} \mathrm{In}_{y} \mathrm{Ga}_{1-x-y} \mathrm{~N}$ have attracted much attention $^{3-5}$ because, unlike in ternary alloys, the band gap and the lattice constant of the quaternary alloy can be individually tuned. For example, it is now possible to have the alloy lattice constant match that of $\mathrm{GaN}$ and simultaneously vary the band gap of the alloy. This is useful in reducing defects, misfit dislocations, and piezoelectric fields in band-gap engineering through, the formation of $\mathrm{Al}_{x} \mathrm{In}_{y} \mathrm{Ga}_{1-x-y} \mathrm{~N} / \mathrm{GaN}$ heterostructures. On the other hand, one can also fix the band gap of the alloy to match that of $\mathrm{GaN}$ or other desired values and vary the alloy composition to improve the structural, optical, and doping properties. Indeed, it has been shown experimentally that photoluminescence (PL) intensity in $\mathrm{Al}_{x} \mathrm{In}_{y} \mathrm{Ga}_{1-x-y} \mathrm{~N}$ quaternary alloys is strongly enhanced compared to that of AlGaN with comparable Al composition. ${ }^{5}$

In this letter, using the first-principles band-structure method and the special quasirandom structure (SQS) approach, ${ }^{6,7}$ we have studied the band structure of $\mathrm{Al}_{x} \mathrm{In}_{y} \mathrm{Ga}_{1-x-y} \mathrm{~N}$ quaternary alloys. We show that the $\mathrm{Al}_{x} \mathrm{In}_{y} \mathrm{Ga}_{1-x-y} \mathrm{~N}$ quaternary alloys can lattice match to $\mathrm{GaN}$ if the ratio between the $\mathrm{Al}$ and In concentrations is approximately $x / y \sim 4.7$. The variation of the band gap for this lattice-matched alloy is about $1.3 \mathrm{eV}$. We also find that when the ratio $x / y \sim 1.8$, the $\mathrm{Al}_{x} \mathrm{In}_{y} \mathrm{Ga}_{1-x-y} \mathrm{~N}$ quaternary alloys will have a band gap similar to that of GaN with a relatively small lattice mismatch $(<1.8 \%)$ between the alloy and $\mathrm{GaN}$. More importantly, we show that the valence band maximum (VBM) and the conduction band minimum (CBM) wave functions of the random quaternary alloy are strongly localized on the InN site, which explains why the PL intensity in

\footnotetext{
${ }^{a)}$ Electronic mail: suhuai_wei@nrel.gov
}

the quaternary alloy is enhanced. As a result of the wavefunction localization, the VBM of the alloy with the same band gap as GaN has a higher VBM than GaN, suggesting that the $p$-type doping in the quaternary alloy should be relatively easier than that in $\mathrm{GaN}^{8-10}$

The band structure and total energy calculations are performed using the density functional theory in the local density approximation ${ }^{11}$ (LDA) as implemented in the planewave VASP code. $^{12-14}$ The Ga $3 d$ and In $4 d$ electrons were explicitly treated as valence electrons. An energy cutoff of $400 \mathrm{eV}$ was used in all cases. For the Brillouin zone integration, we used the $k$-point meshes that are equivalent to the $4 \times 4 \times 4$ Monkhorst-Pack $k$-point meshes ${ }^{15}$ in the zincblende Brillouin zone.

The valence band offset $\Delta E_{v}$ between compound $A X$ and $B Y$ is calculated using an approach that takes into account the deformation potentials of the core reference levels. ${ }^{16}$ In this approach, we first calculate the valence band offset between $A X$ and $B Y$ at their average lattice constant $\bar{a}$ using the standard approach as described in Ref. 17. The contribution of the VBM deformation are then added using the calculated absolute deformation potentials. ${ }^{16}$

To calculate the alloy band gap bowing parameters, we used the more efficient SQS approach. ${ }^{6,7}$ In this SQS approach, instead of occupying the mixed-atom sites of a huge unit cell randomly to gain statistical significance, a relatively smaller unit cell is used, in which the mixed-atom sites of an $A_{1-x} B_{x} C$ alloy are occupied in a way that the physically most relevant structural correlation functions $\bar{\Pi}_{k, m}$ for atomic clusters $(k, m)$ with $k$ vertices, and up to the $m$ th neighbor, are closest to the exact values in an infinite random alloy $\bar{\Pi}_{k, m}=(2 x-1)^{k}$. In this work, we used the $(2 a \times 2 a \times 2 a) 64$ atoms SQS at $x=0.5$. All the atoms inside the cell are fully relaxed by minimizing the quantum mechanical force. For computational convenience, our calculations are performed for cubic zinc-blende (ZB) nitride alloys. Because the band structure of the $\mathrm{ZB}$ and wurtzite (WZ) compounds are very similar near the band edge at $\Gamma$, our results for the ZB alloy are also applicable for the ground-state WZ alloys. 
TABLE I. Calculated equilibrium lattice constant $a$, bulk modulus $B$, and direct band gap at $\Gamma E_{g}$ for AlN, InN, and $\mathrm{GaN}$ in the zinc-blende phase. The calculated values are compared with the experimental values. ${ }^{1,18}$

\begin{tabular}{llll}
\hline \hline & \multicolumn{1}{c}{ AlN } & \multicolumn{1}{c}{ GaN } & \multicolumn{1}{c}{ InN } \\
\hline$a(\AA)$ & $4.335(4.38)$ & $4.445(4.50)$ & $4.961(4.98)$ \\
$B(\mathrm{Mbar})$ & $2.034(2.036)$ & $1.950(2.080)$ & $1.395(1.457)$ \\
$E_{g}(\mathrm{eV})$ & $4.49(6.0)$ & $2.03(3.3)$ & $-0.39(0.7)$ \\
\hline \hline
\end{tabular}

Table I presents our calculated equilibrium lattice constant $a$, bulk modulus $B$, and band gaps at the $\Gamma$ point for zinc-blende phase AlN, GaN, and $\mathrm{InN}$. These results are compared with experimental values (in parentheses). ${ }^{1,18}$ Our calculated lattice parameters $a_{\mathrm{AIN}}, a_{\mathrm{GaN}}$, and $a_{\mathrm{InN}}$ are 4.335 , 4.445, and $4.961 \AA$, respectively, which are in good agreement with the experimental values $4.38,4.50$, and $4.98 \AA$. For the alloys, we find that the Vegard law ${ }^{19}$ is well obeyed; i.e., the lattice constant of the $\mathrm{Al}_{x} \operatorname{In}_{y} \mathrm{Ga}_{1-x-y} \mathrm{~N}$ alloy can be described by

$$
a(x, y)=(1-x-y) a_{\mathrm{GaN}}+x a_{\mathrm{AlN}}+y a_{\mathrm{InN}} .
$$

Using our calculated lattice parameters, we find that the constraint of the alloys lattice matched to GaN implies that $x / y \sim 4.7$, which is in good agreement with the experimental results that the $\mathrm{Al} / \mathrm{In}$ mole fraction ratio is $\sim 4.8$ for wurtzite alloys. $^{5}$

As in most semiconductor alloys, the band gap of the $\mathrm{Al}_{x} \mathrm{In}_{y} \mathrm{Ga}_{1-x-y} \mathrm{~N}$ alloy is a function of concentrations $x$ and $y$ and can be described by an expansion up to the second order in $x$ and $y$,

$$
\begin{aligned}
E_{g}(x, y)= & (1-x-y) E_{g}(\mathrm{GaN})+x E_{g}(\mathrm{AlN})+y E_{g}(\mathrm{InN}) \\
& -b_{\mathrm{AlGaN}} x(1-x)-b_{\mathrm{InGaN}} y(1-y)-b_{x y} x y,
\end{aligned}
$$

where $b$ is the so-called bowing coefficient. To determine these parameters, we calculated the band structure of $\mathrm{Al}_{x} \mathrm{In}_{y} \mathrm{Ga}_{1-x-y} \mathrm{~N}$ alloys using SQS at $(x, y)=(0.0,0.5)$, $(0.5,0.0)$, and $(0.5,0.5)$. The parameter $b_{x y}$ is obtained using $b_{x y}=b_{\mathrm{AIInN}}-b_{\mathrm{AlGaN}}-b_{\mathrm{InGaN}}$ under the quadratic approximation. The calculated bowing coefficients are given in Table II. Our calculated results for $b_{\mathrm{AlGaN}}$ and $b_{\mathrm{InGaN}}$ are 0.688 and $1.416 \mathrm{eV}$, respectively, which are similar to those obtained by Marques et al. ${ }^{4} b_{\mathrm{AlGaN}}=0.632 \mathrm{eV}$ and $b_{\mathrm{InGaN}}=1.370 \mathrm{eV}$, and those recommended by Vurgaftman and Meyer, ${ }^{1} b_{\mathrm{AlGaN}}$ $=0.7 \mathrm{eV}$ and $b_{\text {InGaN }}=1.4 \mathrm{eV}$. For the AlInN alloy, our calculated value $b_{\mathrm{AIInN}}=3.477 \mathrm{eV}$ is larger than the value of $b_{\text {AlInN }}=2.915 \mathrm{eV}$ by Marques et al. which was obtained using the generalized quasichemical approximation (GQCA), and the recommended value of $b_{\mathrm{AlInN}}=2.5 \mathrm{eV}$ by Vurgaftman and Meyer, but it is close to the recent experimental

TABLE II. Calculated bowing parameters $b$ for AlGaN, InGaN, and AlInN alloys. The results are compared with some previous calculations.

\begin{tabular}{lll}
\hline \hline & Present work & Other work \\
\hline$b_{\text {AlGaN }}(\mathrm{eV})$ & 0.688 & $0.632,{ }^{\mathrm{a}} 0.7^{\mathrm{b}}$ \\
$b_{\text {InGaN }}(\mathrm{eV})$ & 1.416 & $1.370,{ }^{\mathrm{a}} 1.4^{\mathrm{b}}$ \\
$b_{\text {AIInN }}(\mathrm{eV})$ & 3.477 & $2.915,{ }^{\mathrm{a}} 2.5,{ }^{\mathrm{b}} 4.96^{\mathrm{c}}$ \\
$b_{x y}(\mathrm{eV})$ & 1.373 & \\
\hline \hline
\end{tabular}

${ }^{\mathrm{a}}$ Reference 4.

${ }^{\mathrm{b}}$ Reference 1 .

${ }^{\mathrm{c}}$ Reference 20 . Reference 20.
Downloaded 12 Jul 2010 to 129.118.86.45. Redistribution subject to AIP license

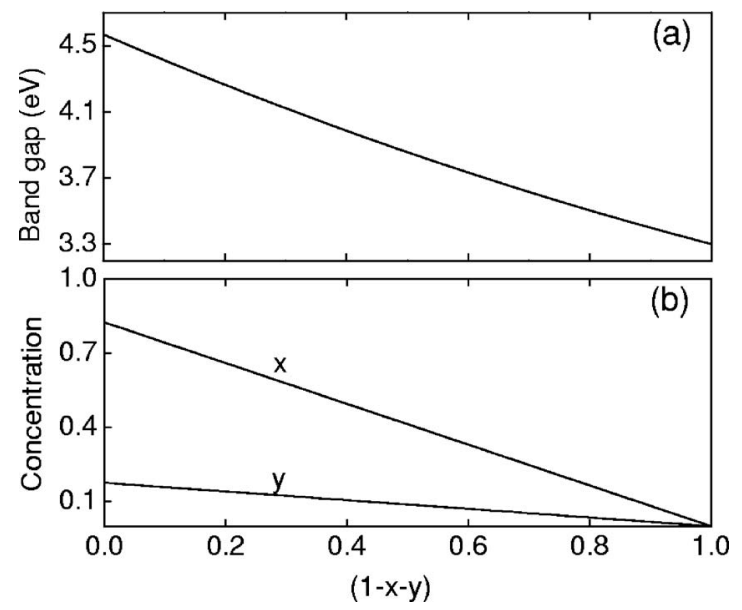

FIG. 1. (a) Band gap of the $\mathrm{Al}_{x} \mathrm{In}_{y} \mathrm{Ga}_{1-x-y} \mathrm{~N}$ alloy as a function of the Ga composition under the constraint that the alloy lattice constant matches that of $\mathrm{GaN}$. (b) The corresponding $\mathrm{Al}$ concentration $x$ and In concentration $y$.

results of $b_{\mathrm{AIInN}}=4.96 \pm 0.28 \mathrm{eV}$ by Terashima et al. ${ }^{20}$

Figure 1 shows the variation of the band gap as a function of $\mathrm{Ga}$ concentration when the lattice constant of the alloy is constrained to that of GaN, i.e., $x / y=4$.7. Because LDA underestimates the band gap, we have adopted the experimental band gaps for the binary zinc-blende compounds (6.0, 0.7, and $3.3 \mathrm{eV}$ for $\mathrm{AlN}, \mathrm{InN}$, and $\mathrm{GaN}$, respectively). Note that because the bowing parameters are obtained using the band-gap differences, the LDA band-gap errors are largely cancelled in the calculation of bowing parameters. We see that under this lattice-matched condition, the band gap can be tuned from $3.30 \mathrm{eV}(x=0, y=0)$ to $4.57 \mathrm{eV}$ $(x=0.825, y=0.175)$, which would be useful for band-gap engineering.

Figure 2 shows the concentration and lattice constant of the alloy when the band gap of the alloy is constrained to match that of GaN. These results are obtained by solving Eqs. (1) and (2). We find that to a good approximation, the ratio of $\mathrm{Al}$ and In concentrations that satisfies this condition is $x / y=1.8$. In the whole range of $\mathrm{Ga}$ concentration, the variation of the lattice constant is less than $1.8 \%$. This indicates that good epitaxial growth of the alloy on GaN substrate is still possible under this band-gap-matched condition.

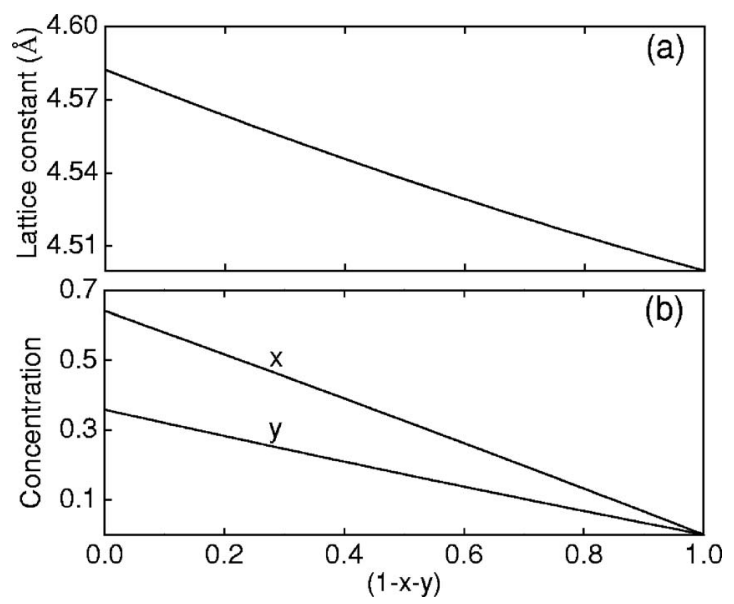

FIG. 2. (a) Lattice constant of $\mathrm{Al}_{x} \mathrm{In}_{y} \mathrm{Ga}_{1-x-y} \mathrm{~N}$ alloy as a function of $\mathrm{Ga}$ composition under the constraint that the alloy band gap is the same as that of $\mathrm{GaN}\left(E_{g}=3.3 \mathrm{eV}\right)$. (b) The corresponding Al concentration $x$ and In con- 


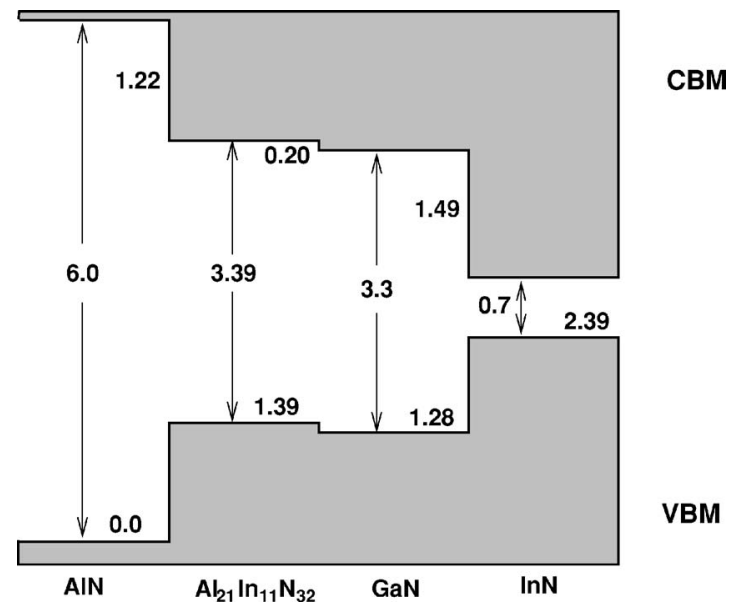

FIG. 3. Band alignment between the binary nitrides and the AlInN alloy that has similar band gap as $\mathrm{GaN}$.

To see how the formation of the quaternary alloy changes the band edge wave function character, we have calculated the valence band offset between $\mathrm{GaN}$ and the alloy that has band gap similar to GaN. Figure 3 shows the calculated results using the method discussed above. We see that because of large volume deformation and $p-d$ repulsion, ${ }^{16}$ the $\mathrm{VBM}$ of $\mathrm{GaN}$ is $1.28 \mathrm{eV}$ higher than that of AlN, and the VBM of InN is $1.11 \mathrm{eV}$ higher than that of $\mathrm{GaN}$. If we assume that the VBM changes linearly as a function of the alloy concentration, one would expect that the VBM of the $\mathrm{Al}_{21} \mathrm{In}_{11} \mathrm{~N}_{32}(x / y=1.9)$ would have a VBM of about $0.46 \mathrm{eV}$ below the VBM of GaN. However, our direct calculations indicate that the calculated VBM of the $\mathrm{Al}_{21} \mathrm{In}_{11} \mathrm{~N}_{32}$ alloy is actually $0.11 \mathrm{eV}$ above that of $\mathrm{GaN}$. This is because in the alloy, both the VBM and CBM states become more localized on the In and neighboring $\mathrm{N}$ sites. For example, for the $\mathrm{Al}_{8} \mathrm{In}_{4} \mathrm{Ga}_{20} \mathrm{~N}_{32}$ alloy, integrations of the charge around each atoms show that the charge around the $\mathrm{InN}$ site is about three times more than the averaged $\mathrm{Ga}$ and $\mathrm{Al}$ sites. Because InN has a much higher VBM, the quaternary alloy with the same gap as GaN could have a higher VBM than GaN. Following the doping-limit rule, ${ }^{8}$ which states that semiconductor with a higher VBM can be doped more easily as $p$ type, we expect that the acceptor level (e.g., $\mathrm{Mg}_{\mathrm{Ga}}$ ) should be shallower and the donor compensation should be reduced in the alloy than in GaN. Moreover, this wave function localization increases the overlap of the VBM and CBM wave functions and thus could enhance the optical transition intensity. Indeed our calculation shows that the dipole matrix element squared $\left|\left\langle\psi_{\mathrm{CBM}}|\mathbf{p}| \psi_{\mathrm{VBM}}\right\rangle\right|^{2}$ of the quaternary $\mathrm{Al}_{8} \mathrm{In}_{4} \mathrm{Ga}_{20} \mathrm{~N}_{32}$ alloy is about twice as large as the GaN alloy. Because the PL intensity is proportional to the matrix element squared, this result is consistent with experimental observations. ${ }^{5}$ It is important to point out that our atomistic SQS model assumes that the cation distribution in the alloy is random. That is, this wavefunction localization already exists in the random alloy due to size and chemical differences between InN and GaN or AlN, as reflected in their band offsets. It is not necessary to have In clustering as a precondition of the wave function localization, although In clustering is expected to enhance the effect of wave function localization.

In summary, we have studied the structural and electronic properties of the quaternary AlInGaN alloys using first-principles methods. The calculated parameters could be useful for band-structure engineering when this alloy is considered for optoelectronic device applications. We also find that the VBM of the alloy is higher than $\mathrm{GaN}$ with the same band gap. Thus the alloy is expected to be doped more easily as $p$ type. We also explained that due to the wave function localization, the PL transition intensity in the alloy is higher than that in GaN.

This work was supported by the National Natural Science Foundation of China under Grants No. 60325416, 60521001, and 90301007. Part of the CPU time for this work was supplied by Supercomputing Center, CNIC, CAS. The research at KSU was supported by U.S. Department of Energy under Contract No. DE-FG02-96ER45604. The work at NREL was funded by the U.S. Department of Energy under Contract No. DE-AC36-99GO10337.

${ }^{1}$ I. Vurgaftman and J. R. Meyer, J. Appl. Phys. 94, 3675 (2003).

${ }^{2}$ H. Morkoc, S. Strite, G. B. Gao, M. E. Lin, B. Sverdlov, and M. Burns, J. Appl. Phys. 76, 1363 (1994).

${ }^{3}$ M. Asif Khan, J. W. Yang, G. Simin, R. Gaska, M. S. Shur, H. Zur Loye, G. Tamulaitis, A. Zukauskas, D. J. Smith, D. Chandrasekhar, and R. Bicknell-Tassius, Appl. Phys. Lett. 76, 1161 (2000).

${ }^{4}$ M. Marques, L. K. Teles, L. M. R. Scolfaro, J. R. Leite, J. Furthmuller, and F. Bechstedt, Appl. Phys. Lett. 83, 890 (2003).

${ }^{5}$ C. H. Chen, L. Y. Huang, Y. F. Chen, H. X. Jiang, and J. Y. Lin, Appl. Phys. Lett. 80, 1397 (2002).

${ }^{6}$ A. Zunger, S.-H. Wei, L. G. Ferreira, and J. E. Bernard, Phys. Rev. Lett. 65, 353 (1990).

${ }^{7}$ S.-H. Wei, L. G. Ferreira, J. E. Bernard, and A. Zunger, Phys. Rev. B 42, 9622 (1990).

${ }^{8}$ S. B. Zhang, S.-H. Wei, and A. Zunger, J. Appl. Phys. 83, 3192 (1998).

${ }^{9}$ W. Kim, A. Salvador, A. E. Botchkarev, O. Aktas, S. N. Mohammad, and H. Morkoc, Appl. Phys. Lett. 69, 559 (1996).

${ }^{10}$ C. G. Van de Walle and J. Neugebauer, J. Appl. Phys. 95, 3851 (2004).

${ }^{11}$ W. Kohn and L. J. Sham, Phys. Rev. 140, A1133 (1965).

${ }^{12}$ G. Kresse and J. Furthmuller, Comput. Mater. Sci. 6, 15 (1996); Phys. Rev. B 54, 11169 (1996).

${ }^{13}$ G. Kresse and J. Joubert, Phys. Rev. B 59, 1758 (1999).

${ }^{14}$ P. E. Blochl, Phys. Rev. B 50, 17953 (1994).

${ }^{15}$ H. J. Monkhorst and J. D. Pack, Phys. Rev. B 13, 5188 (1972).

${ }^{16}$ Y.-H. Li, X. G. Gong, and S.-H. Wei, Phys. Rev. B 73, 245206 (2006);

Y.-H. Li, X. G. Gong, J. Li, and S.-H. Wei (unpublished).

${ }^{17}$ S.-H. Wei and A. Zunger, Appl. Phys. Lett. 72, 2011 (1998).

${ }^{18}$ Semiconductors: Data Handbook, 3rd Ed., edited by O. Madelung (Springer, Berlin, 2004).

${ }^{19}$ L. Vegard, Z. Phys. 5, 17 (1921).

${ }^{20} \mathrm{~W}$. Terashima, S. B. Che, Y. Ishitani, and A. Yoshikawa, Jpn. J. Appl. Phys., Part 2 45, L539 (2006). 